\title{
ON A METHOD OF OBTAINING HALF-YEARLY AND QUARTERLY PREMIUMS FROM THE ANNUAL PREMIUM.
}

\section{To the Editor of the Assurance Magazine.}

Sin,-Allow me to draw the attention of your readers to a method of obtaining half-yearly and quarterly premiums from the annual premium. As it is one of much utility to the practical computer, and I do not remember to have met with it in any of the works in use among actuaries, it may not be altogether unworthy of a place in your columns.

The subject has been treated by the late $\mathrm{Mr}$. Orchard in his exceedingly valuable work, Single and Annual Assurance Premiums, where at p. vii. of the Introduction he gives the expression for finding $c_{x}$, the quantity to be added to half the yearly premium to form the half-yearly premium. That expression is as follows:-

$$
\left.\frac{1-d(1}{2}+x\right)\left(r_{x}+\cdot 75-r_{x+1}\right)=\frac{\mathrm{A}_{x}\left(r_{x}+\cdot 75-r_{x+1}\right)}{2}=c_{x}
$$

which, translated into symbols more generally adopted, is as follows:-

$$
\frac{1-d\left(1+a_{x}\right)}{2}\left(\frac{1}{75+a_{x}}-\frac{1}{1+a_{x}}\right)=\frac{A_{x}}{2}\left(\frac{1}{75+a_{x}}-\frac{1}{1+a_{x}}\right)=c_{x} .
$$

The arithmetical operation is a very simple one, and the value of the increment $c_{x}$ is easily obtained with the assistance of a table of reciprocals; but when these results are collected into a tabular form, there is an inconvenience in the use of them which is not altogether unimportant-viz., that before entering the table it is necessary to find from the annual premium the corresponding annnity, which annuity is made the argument of the table of $c_{x}$.

The method now submitted enables us, by a very obvious substitution in one of the terms of the above quoted expressions, to form the values of $c_{x}$ in terms of the annual premium, instead of in terms of the single premium, and to use the annual premium directly as the argument to the tabular results. It is as follows:-

$$
\begin{gathered}
\text { Since } \frac{1-d\left(1+a_{x}\right)}{1+a_{x}}=\pi_{x}=\text { annual premium, } \\
\pi_{x}\left(1+a_{x}\right)=1-d\left(1+a_{x}\right)
\end{gathered}
$$

and substituting the former of these equivalents for the latter, we obtain a more elegant and convenient expression for the required value of $c_{x}$.

The annual premium then being $\frac{\pi_{x}\left(1+a_{x}\right)}{1+a_{x}}=\pi_{x}$, and the annual amount of the half-yearly premium by the usually adopted approximation being $\frac{\pi_{x}\left(1+a_{x}\right)}{.75+a_{x}}=\pi_{x}+c_{x}$, it is required to find the value of their difference, viz., 


$$
\frac{\pi_{x}\left(1+a_{x}\right)}{\cdot 75+a_{x}}-\frac{\pi_{x}\left(1+a_{x}\right)}{1+a_{x}}=c_{x}
$$

Dividing by $\pi_{x}\left(\mathrm{I}+a_{x}\right)$, and again multiplying by that quantity, we obtain

$$
\pi_{x} \cdot\left(\frac{1+a_{x}}{75+a_{x}}-1\right)=\pi_{x}\left(\frac{\left(1+a_{x}\right)-\left(\cdot 75+a_{x}\right)}{\cdot 75+a_{x}}\right)=\pi_{x} \frac{\cdot 25}{75+a_{x}}=c_{x} .
$$

Then the half-yearly premium $=\frac{\pi_{x}+c_{x}}{2}$ will be

$$
\frac{\pi_{x}+\pi_{x}\left(\frac{\cdot 25}{75+a_{x}}\right)}{2}=\frac{\pi_{x}}{2}\left(1+\frac{\cdot 25}{.75+a_{x}}\right) \text {. }
$$

And generally, when the premium is payable $m$ times a year, the addition to the annuity will be $\left(1-\frac{m-1}{2 m}\right)=\frac{m+1}{2 m}$; and substituting this value in the above equation, it becomes

$$
\pi_{x}\left\{\frac{\left(1+a_{x}\right)-\left(\frac{m+1}{2 m}+a_{x}\right)}{\frac{m+1}{2 m}+a_{x}}\right\}=\pi_{x} \frac{\frac{m-1}{2 m}}{\frac{m+1}{2 m}+a_{x}}=c_{x},
$$

and the preminm for the $m$ th portion of a year will be

$$
\frac{\pi_{s}}{m}\left(1+\frac{\frac{m-1}{2 m}}{\frac{m+1}{2 m}+a_{x}}\right)
$$

Reference has been made above to the facility with which the half-yearly values (to which $\mathrm{Mr}$. Orchard confined his table) may be calculated by his method. The one here given may, however, lay claim to attention upon the ground that the arithmetical operation is thereby still further simplified. An example worked by both methods will enable the reader to form his own estimate of the comparative merits of each of them.

Example.-To find the half-yearly premium when the annual premium is $\mathfrak{E} 4 \cdot 100$.

\section{By Mr. Orchard's method.}

To $\pi_{x}=4 \cdot 100$ the corresponding annuity* is $13 \cdot 260$.

Then $13 \cdot 260+\cdot 75=14 \cdot 010$; its reciprocal $\cdot 071377$

$$
13 \cdot 260+1=14 \cdot 260 \quad, \quad \text { " } \frac{.070126}{.001251}
$$

* थ e., The argument of Mr. Orchard's tables. 


$$
\begin{aligned}
& A_{x} \text { corresponding to annuity* } 13 \cdot 260=58 \cdot 466 \\
& .001251 \\
& \text { and } \frac{58 \cdot 466}{2}=\frac{33292}{2502} \text { inverted } \\
& 1126 \\
& 25 \\
& 4 \\
& .03657 \\
& \frac{\pi_{x}}{2}=\frac{4 \cdot 100}{2}=\frac{2050}{2 \cdot 087}
\end{aligned}
$$

By the method above adduced.

To $\pi_{x}=4 \cdot 100$, the corresponding annuity* is $13 \cdot 260$,

$$
\text { and } \begin{aligned}
& \frac{1}{13 \cdot 260+\cdot 75}=\cdot 071377 \text { and } \frac{.071377}{4}+1 \\
&=1 \cdot 01784 \\
& \pi_{x}=\frac{4 \cdot 100}{2}= \frac{502}{2036} \text { inverted } \\
& \frac{51}{2 \cdot 087}
\end{aligned}
$$

I have calculated a table of half-yearly and quarterly premiums, at 3 per cent., by this method; which I think will be found generally useful, being true for all cases given, irrespective of the rate of mortality, and not first requiring the finding of the corresponding annuity, as is the case with Mr. Orchard's table as already referred to. A copy of the table so calculated is snbjoined.

$$
\begin{aligned}
& \text { I am, Sir, } \\
& \text { Your obedient servant, }
\end{aligned}
$$

$$
\begin{aligned}
& \text { Eagle Life Office, } \\
& \text { Dec. } 1,1863 .
\end{aligned}
$$

SAMUEL L. LAUNDY.

See Note, ante, p. 233. 
Table of Half-yearly and Quarterly Premiums, at 3 per Cent., for any Rate of Mortality, derived from the Annual Premiums by means of the expression

$$
\frac{\pi_{x}}{m} \cdot\left(1+\frac{\frac{m-1}{2 m}}{\frac{m+1}{2 m}+a_{x}}\right) .
$$

\begin{tabular}{|c|c|c|c|c|c|c|c|c|c|c|c|c|c|c|}
\hline Yearly. & $\begin{array}{c}\text { Half- } \\
\text { yearly }\end{array}$ & $\begin{array}{l}\text { Quar- } \\
\text { terly. }\end{array}$ & Yearly. & Half- & $\begin{array}{l}\text { Quar- } \\
\text { terly. }\end{array}$ & Yearly & $\begin{array}{c}\text { Half- } \\
\text { yearly. }\end{array}$ & $\begin{array}{l}\text { Quar- } \\
\text { terly. }\end{array}$ & Yearly. & $\begin{array}{c}\text { Half- } \\
\text { yearly. }\end{array}$ & $\begin{array}{l}\text { Quar- } \\
\text { teriy. }\end{array}$ & \multicolumn{3}{|c|}{ Proportional Parts. } \\
\hline $1 \cdot 00$ & 0.505 & 0.254 & $3 \cdot 30$ & $1 \cdot 676$ & $0 \cdot 845$ & $5 \cdot 60$ & 2861 & $1 \cdot 446$ & $7 \cdot 90$ & $4 \cdot 060$ & $2 \cdot 058$ & $\Delta$ & 13 & 25 \\
\hline$\cdot 05$ & 530 & $\cdot 266$ & $\cdot 35$ & $\cdot 702$ & $\cdot 858$ & .65 & .887 & $\cdot 459$ & .95 & .086 & .072 & & & \\
\hline$\cdot 10$ & .556 & $\cdot 279$ & $\cdot 40$ & $\cdot 727$ & $\cdot 871$ & $\cdot 70$ & 913 & •473 & $8 \cdot 00$ & $\cdot 112$ & 085 & & & \\
\hline$\cdot 15$ & .581 & .292 & $\cdot 45$ & 753 & •884 & $\cdot 75$ & .939 & • 486 & $\cdot 05$ & -138 & $\cdot 099$ & & & \\
\hline 20 & .606 & $\cdot 305$ & $\cdot 50$ & $\cdot 779$ & •897| & 80 & $\cdot 965$ & · 499 & $\cdot 10$ & $\cdot 165$ & •112 & 2 & & 1,0 \\
\hline$\cdot 25$ & .632 & •317 & .55 & $\cdot 804$ & $\cdot 910$ & -85 & •991 & 512 & $\cdot 15$ & $\cdot 191$ & $\cdot 126$ & 3 & 8 & , \\
\hline$\cdot 30$ & $\cdot 657$ & $\cdot 330$ & 60 & •830 & .923 & .90 & 3016 & •525 & $\cdot 20$ & 217 & $\cdot 139$ & 4 & 1,0 & 2,0 \\
\hline$\cdot 35$ & $\cdot 682$ & $\cdot 343$ & $\cdot 65$ & 855 & •936 & 95 & .042 & ·539 & $\cdot 25$ & -243 & ·153 & $\begin{array}{l}4 \\
5\end{array}$ & 1,3 & 40 \\
\hline$\cdot 40$ & 708 & $\cdot 356$ & 70 & $• 881$ & $\cdot 949$ & $6 \cdot 00$ & $• 068$ & •552 & 30 & $\cdot 270$ & $\cdot 166$ & $\begin{array}{l}0 \\
6\end{array}$ & $\begin{array}{l}1,0 \\
1,6\end{array}$ & 3,0 \\
\hline$\cdot 45$ & $\cdot 733$ & •369 & 75 & $\cdot 907$ & •962 & $\bullet 05$ & $\cdot 094$ & $\cdot 565$ & $\cdot 35$ & $\cdot 296$ & $\cdot 180$ & $\begin{array}{l}7 \\
7\end{array}$ & 1,8 & $\begin{array}{l}3,0 \\
3,5\end{array}$ \\
\hline$\cdot 50$ & 758 & •381 & $\cdot 80$ & $\cdot 932$ & $\cdot 975$ & $\cdot 10$ & $\cdot 120$ & •578 & $\cdot 40$ & •322 & $\cdot 193$ & 8 & 2,1 & $\begin{array}{l}4,0 \\
4,0\end{array}$ \\
\hline$\cdot 55$ & $\cdot 784$ & $\cdot 394$ & $\cdot 85$ & $\cdot 958$ & •988 & $\cdot 15$ & $\cdot 146$ & $\cdot 592$ & $\cdot 45$ & $\cdot 349$ & 207 & $\begin{array}{l}0 \\
9 \\
\end{array}$ & & \\
\hline$\cdot 60$ & ·809 & $\cdot 407$ & $\cdot 90$ & •984 & $|\mathrm{I} \cdot 00 \mathrm{I}|$ & -20 & -172 & $\cdot 605$ & 50 & -375 & .220 & & & \\
\hline$\cdot 65$ & $\cdot 834$ & • 420 & $\cdot 95$ & $2 \cdot 009$ & •014 & 25 & $\cdot 198$ & -618 & $\cdot 55$ & $\cdot 401$ & $\cdot 234$ & & & \\
\hline$\cdot 70$ & 860 & - 433 & 4.00 & $\cdot 035$ & $\cdot 027$ & $\cdot 30$ & $\cdot 224$ & •631 & $\cdot 60$ & -427 & $\cdot 247$ & & & \\
\hline$\cdot 75$ & $\cdot 885$ & $\cdot 445$ & $\cdot 05$ & $\cdot 061$ & $\cdot 040$ & $\cdot 35$ & 250 & -645 & $\cdot 65$ & $\cdot 454$ & $\cdot 261$ & $\Delta$ & 14 & 26 \\
\hline$\cdot 80$ & •91] & $\cdot 458$ & $\cdot 10$ & $\cdot 087$ & $\cdot 053$ & $\cdot 40$ & $\cdot 276$ & $\cdot 658$ & $\cdot 70$ & •480 & .274 & & & \\
\hline .85 & .936 & $\cdot 471$ & $\cdot 15$ & $\cdot 112$ & $\cdot 066$ & $\cdot 45$ & $\cdot 302$ & $\cdot 671$ & $\cdot 75$ & $\cdot 506$ & -288 & & & \\
\hline$\cdot 90$ & -962 & $\cdot 484$ & $\cdot 20$ & ·138 & $\cdot 079$ & $\cdot 50$ & •328 & $\cdot 684$ & $\cdot 80$ & 533 & $\cdot 301$ & .001 & $\cdot 000,3$ & $\cdot 000,5$ \\
\hline .95 & •987 & $• 497$ & $\cdot 25$ & $\cdot 164$ & $\cdot 09$ & $\cdot 55$ & $\cdot 354$ & $\cdot 698$ & $\cdot 85$ & .559 & $\cdot 315$ & 2 &, 6 & 1, \\
\hline $2 \cdot 00$ & 1.012 & $\cdot 509$ & $\cdot 30$ & $\cdot 189$ & -105 & $\cdot 60$ & $\cdot 380$ & 711 & .90 & $\cdot 585$ & $\cdot 328$ & 3 & 8 & 1 , \\
\hline$\cdot 05$ & $\cdot 038$ & $\cdot 522$ & $\cdot 35$ & $\cdot 215$ & $\cdot 118$ & .65 & $\cdot 406$ & $\cdot 724$ & $\cdot 95$ & $\cdot 612$ & $\cdot 342$ & 4 & 1,1 & 2 \\
\hline$\cdot 10$ & $\cdot 063$ & $\cdot 535$ & $\cdot 40$ & $\cdot 241$ & -131 & .70 & $\cdot 432$ & $\cdot 738$ & $9 \cdot 00$ & $\cdot 638$ & -355 & 5 & 1,4 & 2 \\
\hline$\cdot 15$ & $\cdot 089$ & $\cdot 548$ & .45 & $\cdot 267$ & $\cdot 144$ & $\cdot 75$ & $\cdot 459$ & $\cdot 751$ & $\cdot 05$ & $\cdot 664$ & $\cdot 369$ & 6 & 1,7 & 3 , \\
\hline$\cdot 20$ & ·114 & $• 561$ & $\cdot 50$ & $\cdot 292$ & $\cdot 157$ & .80 & $\cdot 485$ & $\cdot 764$ & -10 & $\cdot 691$ & $\cdot 382$ & 7 & 2,0 & 3 , \\
\hline 25 & $\cdot 140$ & -574 & $\cdot 55$ & $\cdot 318$ & - 170 & $\cdot 85$ & $\cdot 511$ & $\cdot 778$ & $\cdot 15$ & 717 & ·396 & 8 & 2,2 & 4, \\
\hline$\cdot 30$ & $\cdot 165$ & -586 & $\cdot 60$ & $\cdot 344$ & $\cdot 183$ & -90 & $\cdot 537$ & •791 & $\cdot 20$ & 744 & •409 & 9 & 2,5 & 4 , \\
\hline$\cdot 35$ & $\cdot 191$ & .59 & $\cdot 65$ & $\cdot 370$ & -196 & .95 & $\cdot 563$ & $\cdot 804$ & $\cdot 25$ & 770 & • 423 & & & \\
\hline$\cdot 40$ & .216 & $\cdot 612$ & 70 & $\cdot 396$ & 210 & $7 \cdot 00$ & $\cdot 589$ & $\cdot 818$ & $\cdot 30$ & 796 & $\cdot 437$ & & & \\
\hline$\cdot 45$ & 242 & $\cdot 625$ & $\cdot 75$ & $\cdot 421$ & $\cdot 223$ & $\bullet 05$ & $\cdot 615$ & .831 & $\cdot 35$ & $\cdot 823$ & $\cdot 450$ & $\Delta$ & 15 & 27 \\
\hline$\cdot 50$ & 267 & 6 & $\cdot 80$ & $\cdot 447$ & -236 & $\cdot 10$ & $\cdot 641$ & $\cdot 844$ & $\cdot 40$ & $\cdot 849$ & $\cdot 464$ & $\Delta$ & 10 & 28 \\
\hline .55 & 293 & $\cdot 651$ & -85 & $\cdot 473$ & $\cdot 249$ & -15 & $\cdot 667$ & .858 & $\cdot 45$ & $• 876$ & $\cdot 477$ & & & \\
\hline$\cdot 60$ & $\cdot 318$ & $\cdot 664$ & .90 & • 499 & $\cdot 262$ & $\cdot 20$ & $\cdot 693$ & .871 & $\cdot 5$ & .902 & $\cdot 491$ & & & \\
\hline$\cdot 65$ & 344 & $\cdot 677$ & .95 & 525 & $\cdot 275$ & $\cdot 25$ & $\cdot 720$ & $\cdot 884$ & $\cdot 5$ & .929 & $\cdot 505$ & $\cdot 001$ & 000,3 & $-000,5$ \\
\hline$\cdot 70$ & $\cdot 369$ & $\cdot 690$ & $5 \cdot 00$ & $\cdot 550$ & •288 & $\cdot 30$ & 746 & •898 & $\cdot 6$ & .955 & $\cdot 518$ & 2 & ,6 & I, \\
\hline$\cdot 75$ & $\cdot 395$ & -702 & $\cdot 05$ & 576 & $\cdot 301$ & 35 & $\cdot 772$ & .911 & -65 & •981 & $\cdot 532$ & 3 & ,9 & 1 \\
\hline-80 & $\cdot 420$ & 71 & 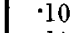 & .602 & ·314 & $\cdot 40$ & $\cdot 798$ & $\cdot 92$ & $\cdot 71$ & 5.008 & $\cdot 545$ & 4 & 1,2 & \\
\hline$\cdot 85$ & $\cdot 446$ & $\cdot 728$ & $\cdot 15$ & $\cdot 628$ & •328 & $\cdot 45$ & $\cdot 824$ & $\cdot 938$ & .75 & $\cdot 034$ & $\cdot 559$ & 5 & 1,5 & 2 \\
\hline$\cdot 90$ & $\cdot 471$ & $\cdot 741$ & $\cdot 20$ & $\cdot 654$ & -341 & 5 & 850 & •951 & 80 & -061 & $\cdot 573$ & 6 & 1, & \\
\hline$\cdot 95$ & $\cdot 497$ & $\cdot 744$ & 25 & $\cdot 680$ & $\cdot 354$ & $\cdot 55$ & .876 & .965 & 85 & $\cdot 087$ & $\cdot 586$ & 7 & 2, & 3 \\
\hline $3 \cdot 00$ & -522 & 76 & $\cdot 30$ & $\cdot 706$ & $\cdot 367$ & $\cdot 60$ & $\cdot 903$ & $\cdot 978$ & $\cdot 90$ & $\cdot 114$ & $\cdot 600$ & 8 & 2 , & 4 \\
\hline 05 & $\cdot 548$ & 780 & $\cdot 35$ & $\cdot 731$ & $\cdot 380$ & .65 & .929 & .991 & $\cdot 95$ & $\cdot 140$ & $\cdot 614$ & 9 & 2,7 & \\
\hline$\cdot 10$ & $\cdot 574$ & 79 & $\cdot 40$ & 757 & •393 & 70 & .955 & 2.005 & $10 \cdot 00$ & $5 \cdot 167$ & • 627 & & & \\
\hline$\cdot 15$ & $\cdot 599$ & 80 & $\cdot 45$ & $\cdot 783$ & $\cdot 40$ & $\cdot 75$ & .981 & $\cdot 018$ & & & & & & \\
\hline 20 & $\cdot 625$ & 819 & $\cdot 50$ & 809 & $\cdot 420$ & $\cdot 80$ & $4 \cdot 007$ & $\cdot 032$ & & & & & & \\
\hline$\cdot 25$ & $\cdot 650$ & $\cdot 832$ & -55 & $\cdot 835$ & $\cdot 433$ & 85 & $\cdot 034$ & $\cdot 045$ & & & & & & \\
\hline
\end{tabular}

